\title{
Enhanced Sensory Properties of a Multichannel Quartz Crystal Microbalance Coated with Polymeric Nanobeads
}

\author{
Simone Pantalei ${ }^{1}{ }^{\text {** }}$, Emiliano Zampetti ${ }^{1}$, Antonella Macagnano ${ }^{1}$, Andrea Bearzotti ${ }^{1}$, \\ Iole Venditti ${ }^{2}$ and Maria V. Russo ${ }^{2}$
}

1 CNR-IMM, Institute for Microelectronics and Microsystems, via del Fosso del Cavaliere 100, 00133 Rome, Italy

2 Department of Chemistry, University of Rome "La Sapienza”, p.le A. Moro 5, 00185 Rome, Italy

* Author to whom correspondence should be addressed. E-mail: simone.pantalei@imm.cnr.it

Received: 1 October 2007 / Accepted: 20 November 2007 / Published: 23 November 2007

\begin{abstract}
In this study the sensorial performances of a four-channel quartz crystal microbalance implemented on a single quartz plate are reported and compared with those of four independent quartz crystal microbalances. Particular attention has been devoted to both cross talk in responses and sensor sensitivity. A recently synthesized nanostructured polymer, poly[phenylacetylene-(co-2-hydroxyethyl methacrylate)], has been used as chemical interactive material. The interactions of our sensor system with relative humidity are also reported. The multichannel device shows a better homogeneity of the mass sensitivity with a spread of the values less then $4 \%$ compared to a $50 \%$ spread observed in the set of four microbalances.
\end{abstract}

Keywords: multichannel quartz crystal microbalance, chemical sensors, nanostructured polymers.

\section{Introduction}

The quartz crystal microbalance (QCM) is one of the widely used chemical sensors in gas sensing for medical, environmental and food applications [1-3]. A fundamental role in chemical sensors is played by the transducer used for transforming the physical-chemical events into electrical signals. Important characteristics like sensitivity and resolution happen to be very influenced by the transducer 
properties. In the present work, a different strategy in the transducer design has been adopted to minimize the spread of the above mentioned characteristics in a set of identically assumed QCMs.

A QCM consists of a single AT-cut quartz crystal oscillating in thickness shear mode. In sensory applications, the couple of electrodes providing the alternating electric field driving the oscillation are typically coated with a chemical interactive material (CIM) able to bind chemically different compounds, both in liquid and in gas phase. The interactions of volatile compounds with CIM induces a frequency decrease in the quartz oscillation proportional to the adsorbed mass, according to the Sauerbrey's equation [4]

$$
\Delta f=-\frac{C_{f} f_{0}^{2}}{A} \cdot \Delta m
$$

where $\Delta f$ is the frequency shift caused by the variation of the adsorbed mass $(\Delta m), C_{f}$ is the mass sensitivity constant, $A$ is the area of the electrodes and $f_{0}$ is the fundamental resonant frequency. The sensor properties, in particular its mass-sensitivity

$$
S=\frac{\partial f}{\partial m}
$$

depend on the chemical characteristics of the CIM coating [5], i.e. its ability to differently adsorb several substances, and on the physical properties of the crystal plate (i.e. the square of the resonant frequency).

Features like sensor reproducibility and accuracy are related to the morphological properties of the coating and to crystal surface. As shown in several works [6,7], the reproducibility in the response of a nominally identical QCM set is strongly dependent on the deposition technique of the CIM. Even tuning the deposition process, the responses of each QCM show a non homogeneous behavior due to the intrinsic differences between each quartz plate. In particular, the different roughness of the quartz plate surfaces caused by lapping and polishing processes could induce a different behavior of the deposited CIM. In fact, the sensor response strongly depends on the CIM active surface area and morphology. Moreover, even slight differences in the cut angle of each quartz plate may induce the QCM set to have different temperature behavior and/or long term stability. In the last years, several studies have shown the possibility to implement sensors based on a multichannel quartz crystal microbalance (MQCM), in which an array of resonators is built on a single quartz crystal plate $[8,9]$. In such a device, an arbitrary $n$ couples of electrodes ( $n$ channels) are deposited on a single quartz plate in order to confine the mechanical oscillations driven by each channel almost completely in a region near the channel itself. In this way, the mass changes relative to a channel produce an oscillation shift not interfering with the other channels. Thus, the channel-to-channel interference and the channel-tochannel mass sensitivity are minimized by depositing the $n$ channels in order to behave as $n$ independent microbalances.

Polymers are widely used to prepare self-assembled nano-structured materials for their greater synthetic flexibility in comparison with inorganic ones (e.g. titania, silica) and for the feasibility of chemical synthesis procedures producing monodisperse nanospheres with controlled dimensions: these 
nanostructured materials find application in optical and electronic devices, sensors and bio-sensors [10]. In sensor technology the nanostructure of these CIMs enhances the capability of gas detection and the dynamic range of the sensor. This is mainly due to both the higher active surface area and its highly ordered morphology [11-13].

\section{Results and Discussion}

In this study the possibility to implement a sensor based on four QCMs laying on the same quartz plate has been exploited in order to minimize the inhomogeneities in CIM behavior depending on the piezoelectric substrate. The sensing unit has been implemented by evaporating four chromium-gold electrodes on both surfaces of a $160 \mu \mathrm{m}$ thickness and $14 \mathrm{~mm}$ diameter quartz plate having a fundamental oscillation frequency of $10 \mathrm{MHz}$. By means of subsequent additive gold evaporations each electrode has shown a different thickness, resulting in distinct oscillation frequencies, always lower than that of the blank quartz. The geometry of each electrode has been tuned in order to keep most of the vibrational energy near to the region of the electrodes (energy trapping) and their area has been taken below the Bechmann's number, so that the responses of the anharmonic overtones are negligible [14]. With this particular design and optimizing the distance between the electrodes [15], it is possible to maintain the channel-to-channel interference at a very low level.

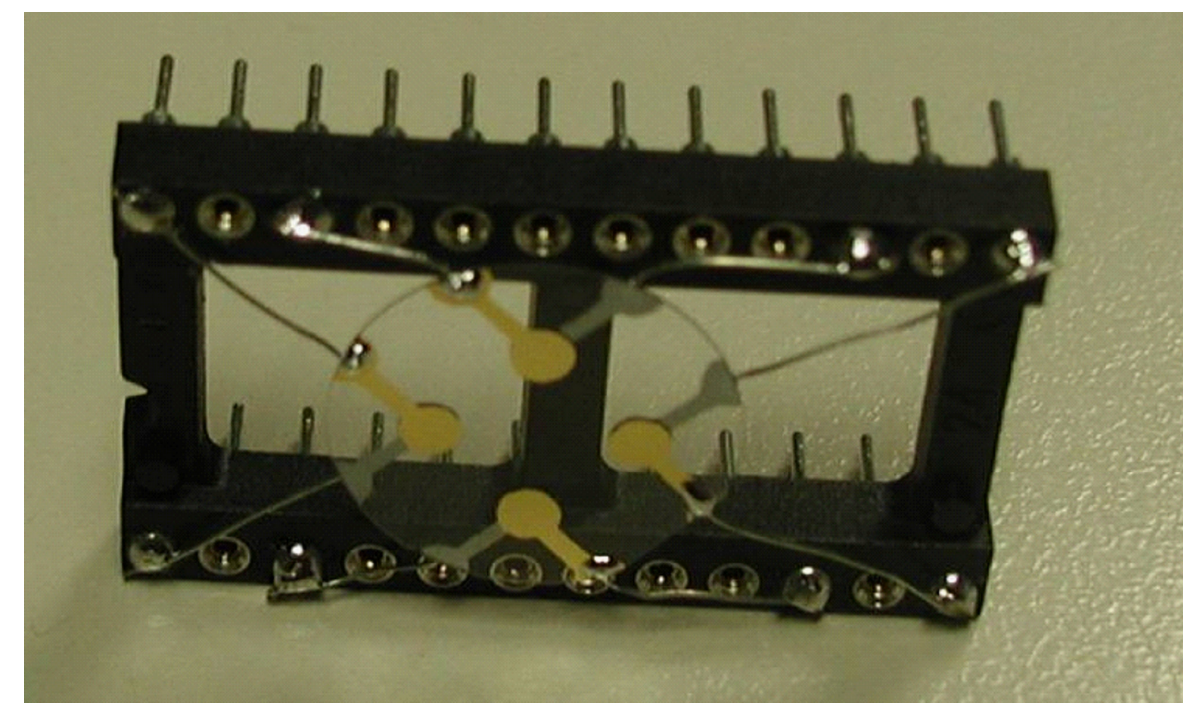

Figure 1. The prototype of a MQCM layed on a single quartz plate used for the electrical characterization.

The implemented MQCM (Figure 1) is a four-ports network, characterized by a scattering matrix, representing the reflected and transmitted powers at each port, in the frequency domain. In this way, the network is fully characterized by knowing the 10 independent coefficients, $\mathrm{S}_{\mathrm{ij}}$.

The outline of the scattering matrix measurements is depicted in Figure 2. The $S_{i j}$ parameters have been measured by a network analyzer (Agilent Technologies E5061A), exciting the $\mathrm{j}^{\text {th }}$ port with a driving power in the frequency range of interest and reading the reflected power on the $i^{\text {th }}$ port. Contemporarily, the remaining two ports have been closed with the internal adapting impedance of the instrument, i.e. $50 \Omega$. 


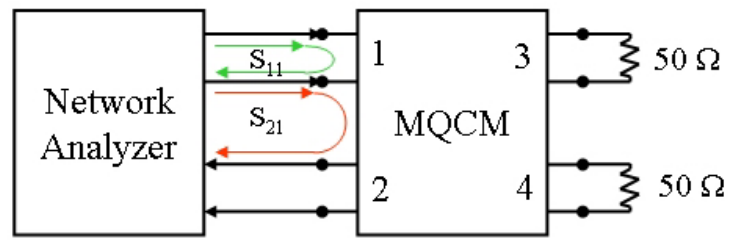

$$
S=\left[\begin{array}{llll}
s_{11} & S_{12} & s_{13} & s_{14} \\
& S_{22} & S_{23} & s_{24} \\
& & S_{33} & s_{34} \\
& & & s_{44}
\end{array}\right]
$$

Figure 2. Outline of the scattering coefficients measurements for the MQCM device, represented as a four-ports network (on the left). The scattering matrix (on the right) is characterized by 10 independent parameters, since the matrix is reciprocal (i.e. $\mathbf{s}_{\mathrm{ij}}=\mathbf{s}_{\mathrm{ji}}$ when $\mathrm{i} \neq \mathrm{j}$ ).

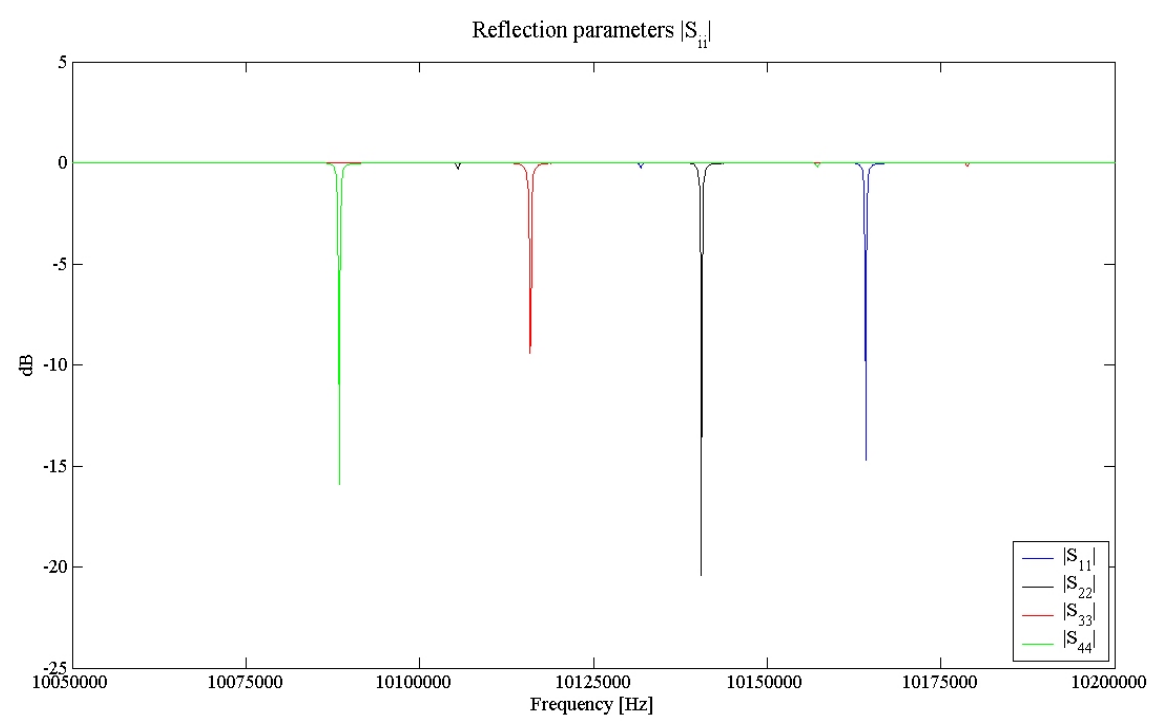

Figure 3. Moduli of the reflection parameters of the four ports network corresponding to the MQCM.

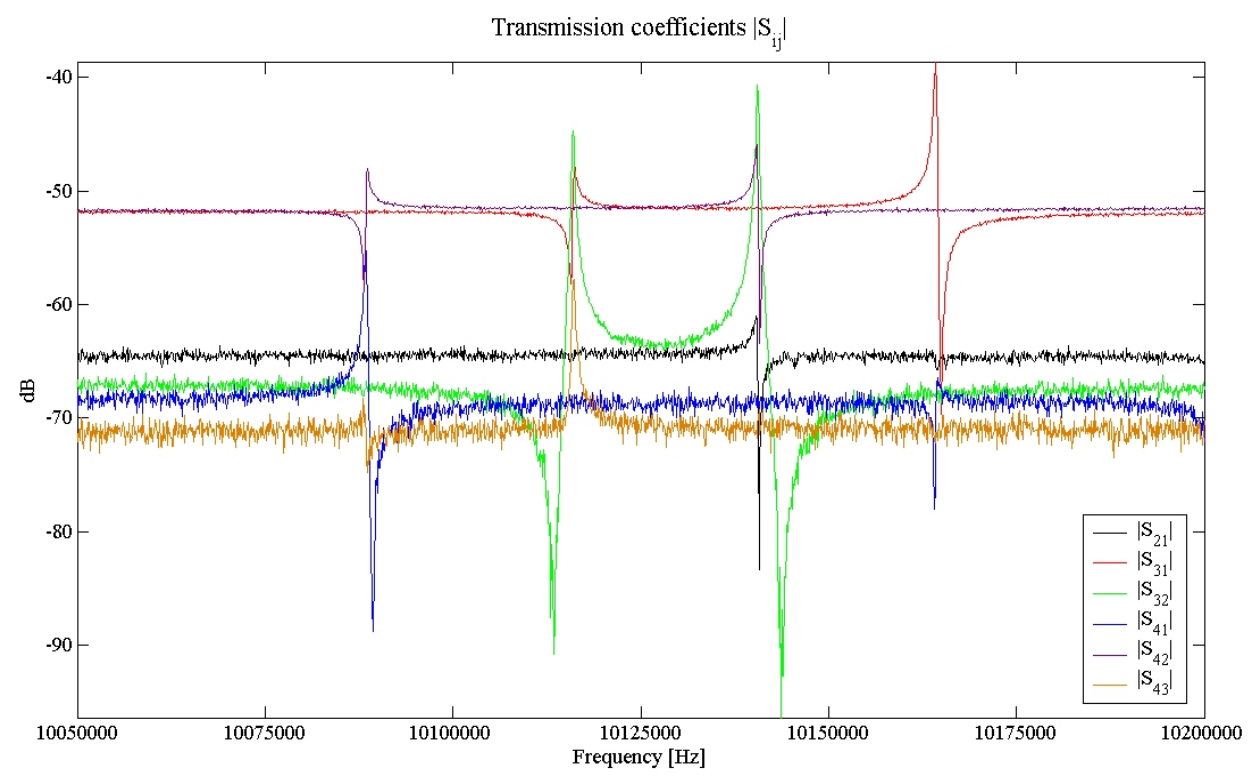

Figure 4. Moduli of the transmission parameters of the four ports network corresponding to the MQCM. 
The resulting scattering parameters are plotted in Figure 3 and 4. The absolute values of the reflection parameters point out the good separation of the four channels in the frequency range of interest, while the moduli of the transmission parameters, showing typical values of less than $-60 \mathrm{~dB}$, indicate the low existing channel-to-channel crosstalk, always less than $-40 \mathrm{~dB}$ [16]. This result ensures that the channels oscillate almost independently on each others.

In order to test the characteristics of the MQCM transducer a chemical sensor has been implemented utilizing a new semiconducting co-polymer, namely poly [phenylacetylene-(co-2hydroxyethyl methacrylate)] (P(PA/HEMA)), synthesized in the form of monodispersed nanospheres. The use of different reaction conditions during the synthesis of this co-polymer allows to modulate dimension, polydispersity and superficial charge of the beads [17]. In Figure 5 it is shown the chemical structure of the P(PA/HEMA) and the morphological aspect of the polymer film as seen at SEM. The use of a polar co-monomer (HEMA), which provides the stability of the nanospheres in water, and a hydrophobic polymer (PA), allows to produce a co-polymer with charged surface and hydroxyl groups on the particles surface. These properties of the nanospheres improve their adhesion on substrates, the high order of wide domains of CIM coating and the capability to bind polar molecules. The resulting chemical sensor has been tested at different relative humidity values.

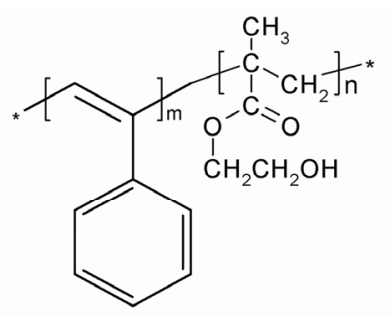

(a)

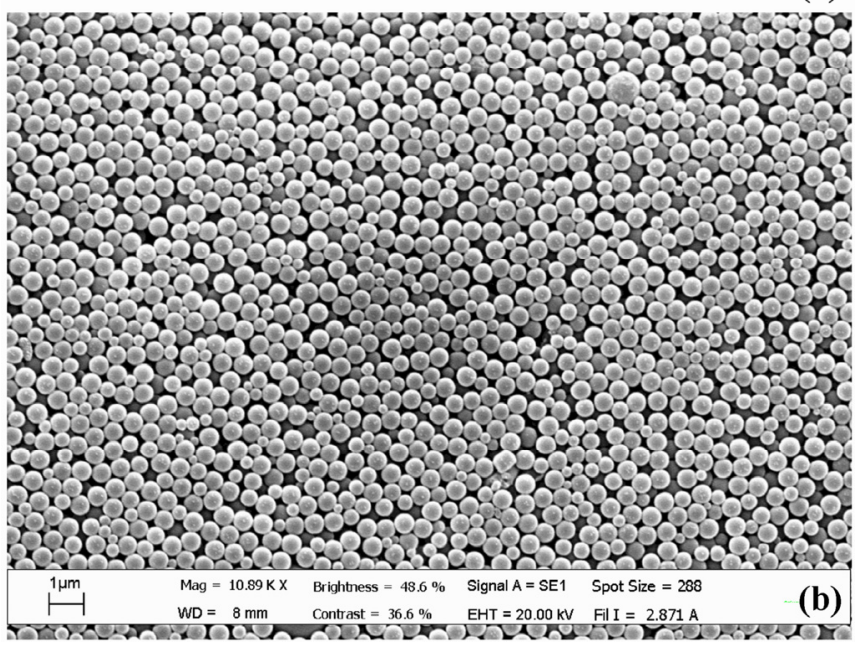

Figure 5. Chemical structure of $\mathrm{P}(\mathrm{PA} / \mathrm{HEMA})$ (a). Morphology of the nanostructured polymer film used as CIM as seen at SEM (b).

To implement the sensing unit, different masses of an aqueous solution of the nano-structured polymer P(PA/HEMA) have been deposited on the four channels of the MQCM and on four single QCM by casting technique. Once the solvent has evaporated, a thin film of the material has remained on the substrate. According to Sauerbrey's equation (1), the different masses of the deposited polymer cause different frequency shifts as shown in Figure 6. 

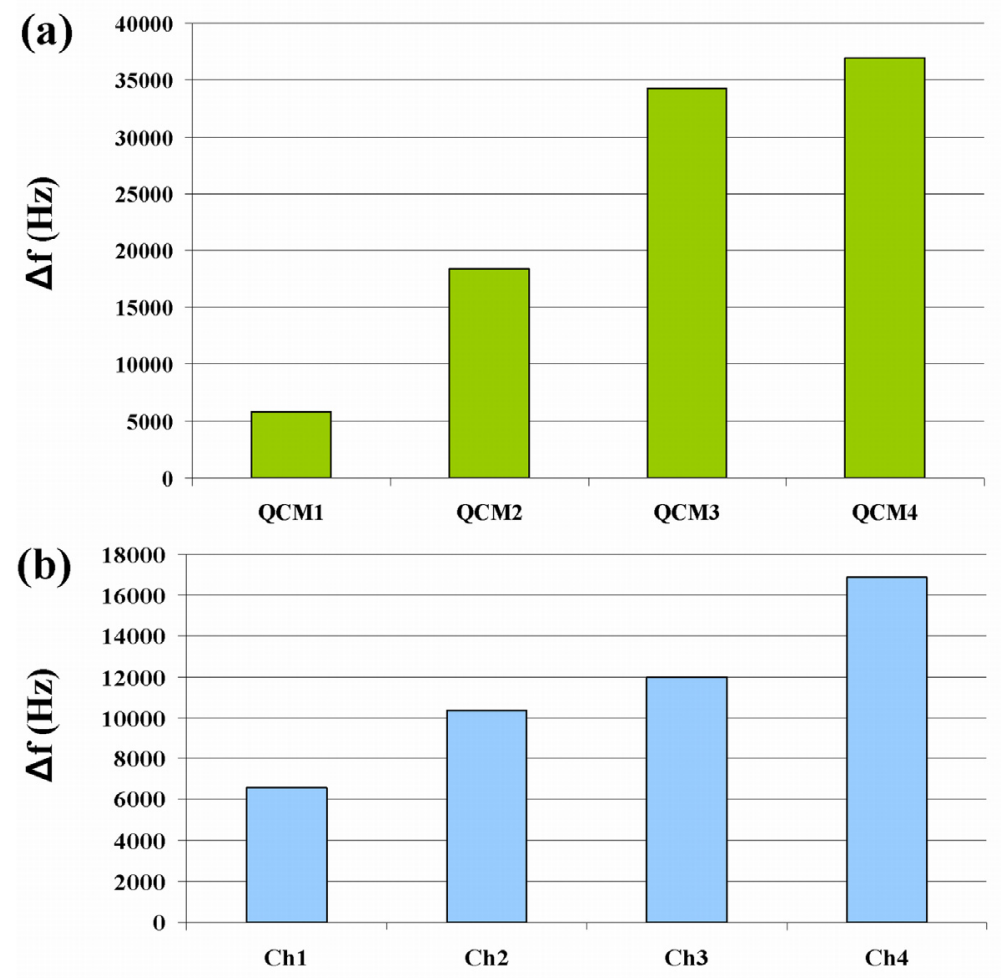

Figure 6. Frequency shift $\left(\Delta f=f-f_{0}\right)$ caused by the CIM deposition onto single QCM sensors (a) and MQCM channels (b). $f$ is the resonant frequency after the deposition and $f_{0}$ is the fundamental resonant frequency.

\section{Experimental}

The measurements have been performed in a sealed chamber, where a mass flow controller system has set the desired relative humidity values by mixing a dry and humid loaded stream of nitrogen. The chamber temperature has been also monitored and recorded. The sensors have been driven by a suitable oscillator circuit, in the modified Colpitts configuration. The output frequency values have been measured with an universal frequency counter (Racal Dana HR 1991, resolution $0.1 \mathrm{~Hz}$ ) and transmitted through IEEE 488 interface to a PC unit. The MQCM sensor and the set of four QCMs have been characterized at different relative humidity values.

In order to better compare the sensitivities of the MQCM to the four QCM sensors, measurements have been performed in a range of $5 \%$ to $50 \%$ of relative humidity. Here the response of the sensors is linear and their sensitivities, as defined in (2), are constant. The dependence of the shift of the resonant frequencies of the sensors, $\Delta f$, on relative humidity variations are depicted in Figure 7 . These values have been measured when the adsorption process has been in steady state condition. The response curves have shown a linear trend, as expected, while the angular coefficients corresponding to the sensitivities of the sensors have been different for both the four QCMs and the MQCM channels. This peculiarity is mainly related to the different mass of $\mathrm{P}(\mathrm{PA} / \mathrm{HEMA})$ deposited on each microbalance and slightly depending on physical characteristics of the quartz plates. In fact, by increasing the mass of the layer, the number of the sites available for the ad-absorption processes increases too [18]. 

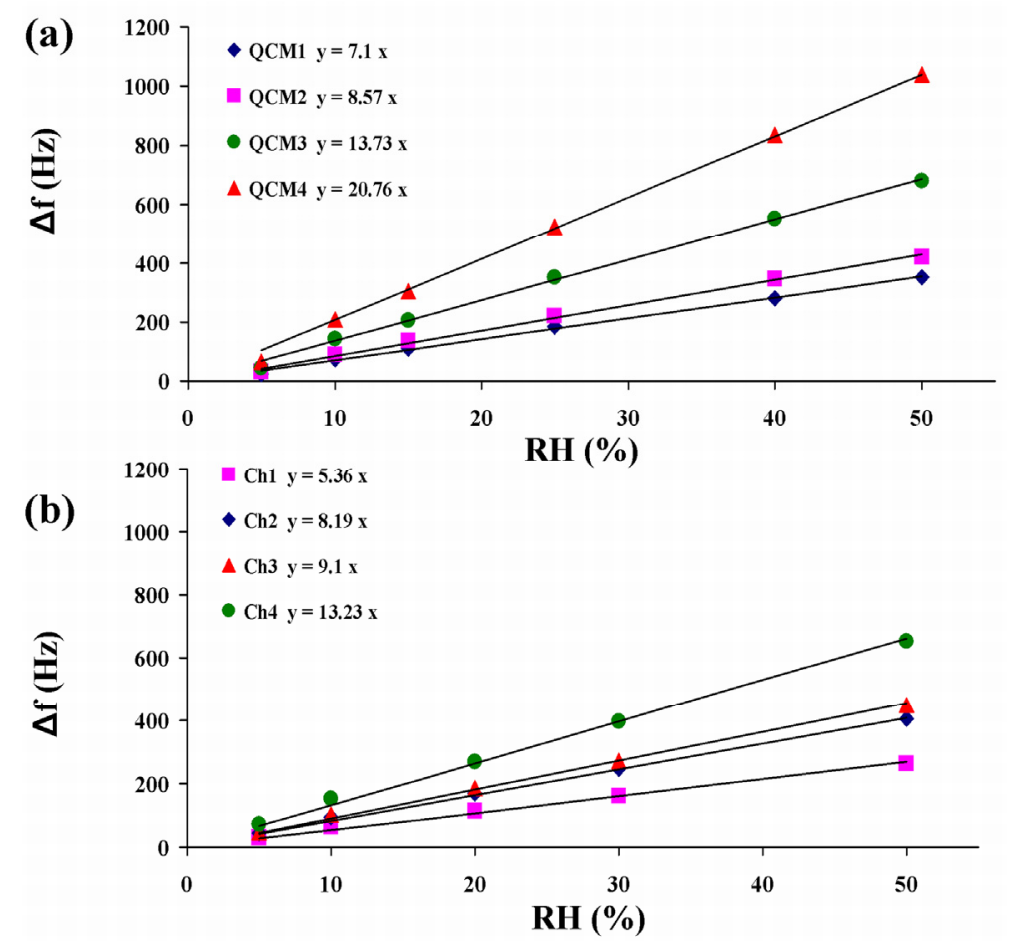

Figure 7. Responses of the QCM sensors (a) and MQCM channels (b) vs. relative humidity variation. Data are fitted by straight lines with angular coefficients representing the sensitivity of each sensor, see (2).

(a)
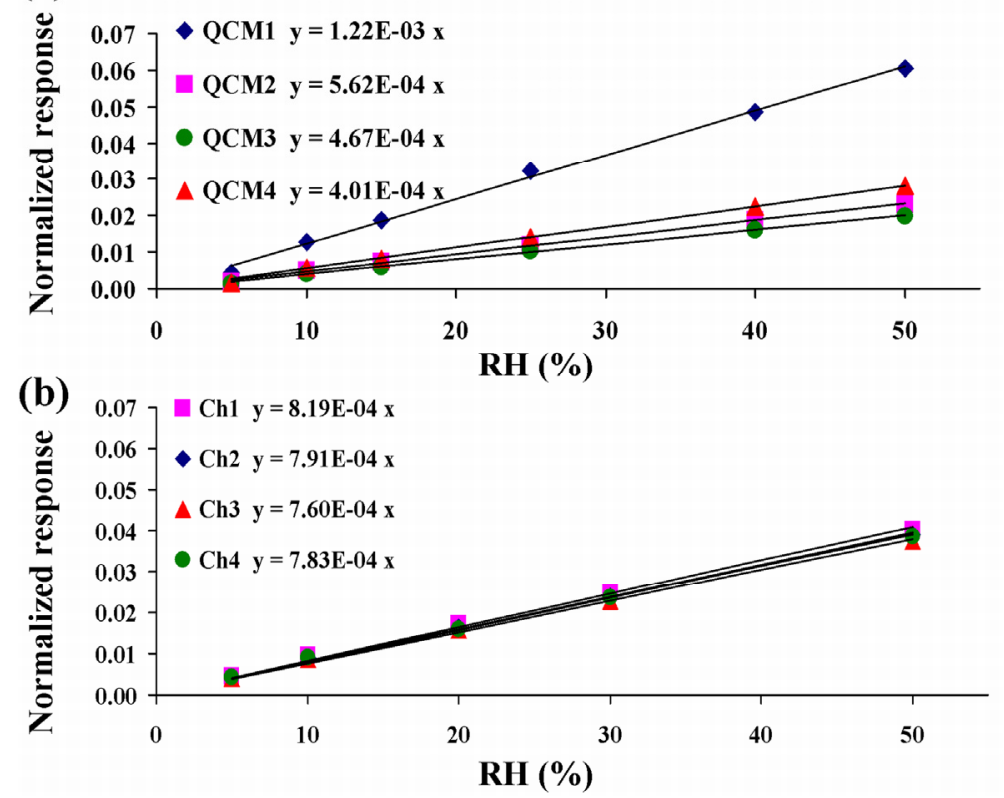

Figure 8. Normalized responses of the QCM sensors (a) and MQCM channels (b) vs. relative humidity variation.

Normalizing each sensor response to its relative deposited mass, i.e. the values of frequency shift, $\Delta f$, caused by the respective mass loading (Figure 6), it is possible to compare the normalized sensitivity for each microbalance (Figure 8). The angular coefficients of the linear fittings represent the normalized sensitivities of the sensors. The MQCM channels (b) show the improved homogeneity in 
the normalized sensitivity compared to the QCM set (a).In the case of the four distinct QCMs the normalized sensitivities range from $4 \cdot 10^{-4}$ to $12 \cdot 10^{-4}(\mathrm{RH} \%)^{-1}$ with a spread of $50 \%$ from the central value. Having removed the contribution of the polymer mass, we can assume that this effect is due to intrinsic differences in the quartz plate. On the other hand the MQCM device shows an extremely good agreement among the normalized sensitivities of different channels, with values ranging from $7.6 \cdot 10^{-4}$ to $8.2 \cdot 10^{-4}\left(\mathrm{RH}^{-1}\right)^{-1}$ and a spread of $3.8 \%$ from the media. This result is presumably attributed to the particular design of the MQCM sensor implemented on a single quartz plate, since the physical characteristics originating from the quartz substrate are the same for each channel.

\section{Conclusions}

A multichannel quartz crystal microbalance consisting of four resonators laid on a single quartz plate has been fabricated. This device has been electrically characterized and has shown hi quality performances in terms of channel-to-channel separation. Subsequently, it has been coated with a new semiconducting polymer, P(PA/HEMA), synthesized in form of nanostructured spheres and then its sensory properties have been compared with those of four separate quartz crystal microbalances. The MQCM exhibits a very good agreement for the values of the normalized mass sensitivity respect to the variations of relative humidity, compared to those of the set of QCMs.

An important feature of the small spread in the sensitivity is an enhancement of the accuracy parameter and an easier calibration procedure. Moreover, problems that could arise during the CIM deposition processes on MQCM transducer are minimized and the performances of the sensor remain substantially unaltered. Finally both reduced dimension, mass and limited power consumption make it appealing in aerospace, automotive and medical applications.

\section{References and Notes}

1. Mecea, V.M. Is quartz crystal microbalance really a mass sensor? Sensors and Actuators A 2006, $128,270-277$.

2. Zhang, J.; Hu, J.Q.; Gong, H.; O'Shea, S.J. Quartz crystal microbalance coated with sol-gelderived thin films as gas sensor for NO detection. Sensors 2003, 3, 404-414.

3. Mannelli, I.; Minunni, M.; Tombelli, S.; Mascini, M. Quartz crystal microbalance (QCM) affinity biosensor for genetically modified organisms (GMOs) detection. Biosensors and Bioelectronics 2003, 18, 129-140.

4. Sauerbrey, G. Verwendung von Schwingquarzen zur Wägung dünner Schichten und zur Mikrowägung. Zeitschrift für Physik 1959, 155, 206-222.

5. Gomes, M.T.S.R.; Duarte, A.C.; Oliveira, J.A.B.P. Critical assessment of the parameters that affect the selection of coating compounds for piezoelectric quartz crystal microbalances. Talanta 1999, 48, 81-89.

6. Gomes, M.T.; Duarte, A.C.; Oliveira, J.P. Comparison of two methods for coating piezoelectric crystals. Analytica Chimica Acta 1995, 300, 329-334.

7. Muñoz-Aguirre, S.; Nakamoto, T., Moriizumi, T. Study of deposition of gas sensing films on quartz crystal microbalance using an ultrasonic atomizer. Sensors and Actuators B 2005, 105, 144 149. 
8. Tatsuma, T.; Watanabe, Y.; Oyama, N.; Kitakizaki, K.; Haba, M. Multichannel Quartz Crystal Microbalance. Analytical Chemistry 1999, 71, 3632-3636.

9. Hung, V.N.; Abe, T.; Minh, P.N.; Esashi, M. Miniaturized, highly sensitive single-chip multichannel quartz-crystal microbalance. Applied Physics Letters 2002, 81, 5069-5071.

10. Mensitieri, G.; Venditto, V.; Guerra, G. Polymeric sensing films absorbing organic guests into a nanoporous host crystalline phase. Sensors and Actuators B 2003, 92, 255-261.

11. Penza, M.; Cassano, G.; Aversa, P.; Antolini, F.; Cusano, A.; Cutolo, A.; Giordano, M.; Nicolais, L. Alchool detection using a carbon nanotubes acoustic sensor. Applied Physics Letters 2004, 85, 2379-2381.

12. Zhang, Z.; Chen, H.; Zhong, J.; Chen, Y.; Lu, Y. ZnO Nanotip-based QCM Biosensors. In Proceedings of the IEEE International Frequency Control Symposium and Exposition, Miami, FL, June 2006; pp. 545-549.

13. Chao, T.W.; Liu, C.J.; Hsieh, A.H.; Chang, H.M.; Huang, Y.S.; Tsai, D.S. Quartz crystal microbalance sensor based on nanostructured $\mathrm{IrO}_{2}$. Sensors and Actuators B 2007, 122, 95-100.

14. Mindlin, R.D.; Lee, P.C.Y. Thickness-shear and flexural vibrations of partially plated crystal plates. International Journal of Solids and Structures 1966, 2, 125-139.

15. Shen, F.; Lu, P. Influence of interchannel spacing on the dynamical properties of multichannel quartz crystal microbalance. IEEE Transaction on Ultrasonic, Ferroelectrics and Frequency Control 2004, 51, 249-253.

16. Pantalei, S.; Zampetti, E.; Macagnano, A.; Martinelli, E.; Paolesse, R.; Di Natale, C.; D’Amico, A. A multichannel quartz based electronic nose. In Proceedings of the EUROSENSORS XX Conference, Göteborg, Sweden, Sept 17-20, 2006; Vol. 2, pp. 416-417.

17. Venditti, I.; D’Amato, R.; Russo, M.V.; Falconieri, M. Synthesis of conjugated polymeric nanobeads for photonic bandgap materials. Sensors and Actuators B 2007, 126, 35-40.

18. Macagnano, A.; Sgreccia, E.; Paolesse, R.; De Cesare, F.; D’Amico, A.; Di Natale, C. Sorption and condensation phenomena of volatile compounds on solid-state metalloporphyrin films. Sensors and Actuators B 2007, 124, 260-268.

(C) 2007 by MDPI (http://www.mdpi.org). Reproduction is permitted for noncommercial purposes. 